\title{
Scoliosis in Children: Impact of Goal Directed Therapies on Intra-operative and Postoperative Outcomes
}

Claudine Kumba ( $\sim$ claudine.kumba@gmail.com )

Hôpital Universitaire Necker Enfants Malades, Assistance Publique Hôpitaux de Paris, APHP, Université de Paris https://orcid.org/0000-0002-9748-5141

Lotfi Miladi

Hôpital Universitaire Necker Enfants Malades, Assistance Publique Hôpitaux de Paris, APHP, Université de Paris

\section{Short Report}

Keywords: Scoliosis, Children, Outcome, Goal Directed Therapies

Posted Date: September 24th, 2021

DOl: https://doi.org/10.21203/rs.3.rs-765785/v2

License: (a) (i) This work is licensed under a Creative Commons Attribution 4.0 International License. Read Full License

Version of Record: A version of this preprint was published at Open Journal of Orthopedics on October 1st, 2021. See the published version at https://doi.org/10.4236/ojo.2021.1110030. 


\section{Abstract}

Background: Scoliosis is among interventions with high postoperative complication rates due to the characteristics of the surgery where blood loss, transfusion and fluid requirements can be increased.

A monocentric retrospective observational study was undertaken earlier to determine predictors of intraoperative and postoperative outcomes in surgical patients. In this initial cohort there were patients who underwent scoliosis surgery and a secondary analysis to describe outcomes in these patients was realized and presented here.

Objective: To describe intraoperative and postoperative outcomes in patients under 18 years old in scoliosis surgery included in the initial study and to propose improvement implementation measures.

Methods: Secondary analysis of patients in scoliosis surgery. The study was approved by the Ethics Committee.

Results: There were 116 patients with a mean age of $147.5 \pm 40.2$ months. Twenty-eight patients $(24.1 \%)$ presented intraoperative and or postoperative complications. The most common intraoperative complication was hemorrhagic shock in 3 patients $(2.6 \%)$. The most common postoperative organ failure was neurologic in seven patients $(6 \%)$, respiratory in 3 patients $(2.6 \%)$, cardio-circulatory in 2 patients $(1.7 \%)$ and renal failure in one patient $(0.9 \%)$.

The most common postoperative infection was surgical wound sepsis in 8 patients $(6.9 \%)$, urinary sepsis in three patients $(2.6 \%)$, abdominal sepsis and septicemia in two patients $(1.7 \%)$ respectively.

Twelve patients (10.3\%) had reoperations.

Fifty-six patients (48.3\%) had intraoperative transfusion.

There was no in-hospital mortality.

Conclusion: Integrating goal directed therapies in this surgical setting could improve postoperative outcomes

\section{Introduction}

Scoliosis surgery is one of the most common performed major elective surgeries in our hospital.

This disorder of the vertebral column is classified as idiopathic or juvenile and non idiopathic scoliosis (see figures 1 and 2 for illustration).

The etiopathology of juvenile scoliosis is unknown whereas non idiopathic scoliosis is associated with neuromuscular diseases, bone diseases or other syndromes. Postoperative morbidity in scoliosis is high due to the characteristics of the surgery where blood losses, transfusion and fluid therapy requirements 
can be increased (1). The patient general status is one of the predictors of postoperative evolution (2). There is growing evidence that applying enhanced recovery after surgery protocols in scoliosis surgery in children reduces postoperative morbidity in terms of organ dysfunction and length of hospital stay (LOS) (3). We conducted earlier in our hospital a monocentric observational study in neurosurgical, abdominal surgical and orthopedic patients to determine predictors of intra-operative and postoperative outcomes (2). In this cohort of 594 patients there were patients who underwent scoliosis surgery. We aimed with this secondary analysis of the initial study to describe intraoperative and postoperative outcomes in patients who had scoliosis surgery and to implement improved intraoperative patient management protocols with the objectives of optimizing postoperative outcome in this surgical population.

\section{Methods And Materials}

Secondary analysis of patients who underwent scoliosis surgery included in the initial study (2).

The study was registered to the National Commission for Computer Science and Liberties (CNIL) under the number 2028257 v0 on 21 February 2017 and approved by the Ethics Committee of our hospital under the registration number 2017-CK-5-R1 on 21 March 2017.

Inclusion criteria were patients included in the initial study aged less than 18 years old and who underwent scoliosis surgery.

Exclusion criteria were patients above 18 years old and who did not undergo scoliosis surgery included in the initial study.

Patients were included retrospectively from 1 January 2014 to 17 May 2017.

Statistics were analyzed with XLSTAT 2020.4.1. software. Continuous variables were expressed as medians with ranges or means with standard deviations. Categorial variables were described in proportions.

In our hospital, scoliosis surgical patients were managed perioperatively according to a protocol described here. Preoperatively patients had respiratory functional tests, iron and erythropoietin supplementation, complete dental examination, nasal antibiotic therapy with mupirocin and a special fiber free diet several days prior to surgery, complete blood cell count and packed red blood cells units available. Intraoperatively 2 large peripheral intravenous lines and an arterial catheter were inserted; patients were monitored with bispectral index, indwelling bladder catheter, nasogastric tubing, central core temperature probe, fluid warming system, warming blanket and somesthesic evoked potentials. Induction of anesthesia could be inhalational with Sevoflurane, or intravenous with Propofol, Remifentanil and Ketamine. Maintenance of anesthesia was intravenous with Propofol, Remifentanil and Ketamine. Airway was secured with orotracheal or nasotracheal intubation. Tranexamic acid was administered as an intravenous bolus of $30 \mathrm{mg} / \mathrm{kg}$ followed by an intravenous infusion of $10 \mathrm{mg} / \mathrm{kg} / \mathrm{h}$. A cell saver was available in case of non-idiopathic scoliosis. Antibiotic prophylaxis was performed with 
cefazolin and or vancomycin depending on patient's microbiological status. Fluid therapy was performed with crystalloids as $10-20 \mathrm{ml} / \mathrm{kg}$ bolus and colloids (Plasmion ${ }^{\circledR}$ ) as $30 \mathrm{ml} / \mathrm{kg}$ bolus.

The objective was to maintain mean arterial pressure above $60 \mathrm{mmHg}$.

Postoperative analgesia was realized with spinal analgesia with morphine as $5 \mu \mathrm{g} / \mathrm{kg}$ (maximum $500 \mu \mathrm{g}$ ) administered intraoperatively, acetaminophen, ketoprofen, clonidine and patient controlled analgesia with intravenous bolus morphine.

Patients were extubated in the operating room or in the recovery room.

\section{Results}

Table 1 illustrates the general characteristics

There were 116 patients with a mean age of $147.5 \pm 40.2$ months and median weight of 34 kilograms [24-45]. 63 patients (54.3\%) were American Society of Anesthesiologists grade 3 (ASA 3), 17 patients $(14.7 \%)$ were ASA 4 . Seventy-one patients $(61.2 \%)$ had neuromuscular or syndromic scoliosis and forty-five patients (38.8\%) had idiopathic scoliosis. 111 patients $(95.7 \%)$ had elective surgery.

Twenty-eight patients (24.1\%) presented intraoperative and or postoperative complications.

The most common intraoperative complication was hemorrhagic shock in 3 patients $(2.6 \%)$ followed by anaphylaxis and respiratory failure in one patient $(0.9 \%)$ respectively. The most common postoperative organ failure was neurologic in seven patients $(6 \%)$ followed by respiratory in 3 patients $(2.6 \%)$, followed by cardio-circulatory in 2 patients $(1.7 \%)$ and renal failure in one patient $(0.9 \%)$.

The most common postoperative infection was surgical wound sepsis in eight patients (6.9\%), followed by urinary sepsis in three patients $(2.6 \%)$, followed by abdominal sepsis and septicemia in two patients $(1.7 \%)$ respectively.

Twelve patients $(10.3 \%)$ had reoperations.

Fifty-six patients (48.3\%) were intraoperatively transfused with packed red blood cells and or fresh frozen plasma and or platelet units.

There was no in-hospital mortality.

Median length of intensive care unit stay was 4[0-25] days. Median length of hospital stay was 10[150] days. Median total length of hospital stay was 12[2-67] days. Median length of mechanical ventilation was $0[0-21]$ days.

Table 2 illustrates co-morbidities. 
The most common co-morbidities were cerebral anoxic lesions in 22 patients (18.9\%), followed by neurofibromatosis in 7 patients (6\%), followed by myelomeningocele, osteogenesis imperfecta and polymalformative syndrome in 4 patients $(3.5 \%)$ respectively, followed by congenital heart disease and psychomotor deficiency in 2 patients $(2.5 \%)$ respectively.

\section{Discussion}

With regards to the rate of patients with intraoperative and or postoperative complications,

including transfusion guided protocols with point of care tests, goal directed fluid and hemodynamic therapy with validated tools and parameters in children and integrating enhanced recovery after surgery protocols to optimize intraoperative management in scoliosis surgery could improve postoperative outcome in this surgical setting $(3,4,5,6,7,8,9,10,11,12,13,14,15)$.

To improve blood product administration practices in scoliosis surgery, transfusion guided protocols with point of care viscoelastic assays should be included in this setting. Point of care tests in hemorrhagic interventions have been shown to reduce fresh frozen plasma administration and length of hospital stay in children (4). Algorithms with rotational thromboelastometry are illustrated in figures 3 to 5 to guide transfusion in hemorrhagic surgery (15). Intraoperative goal directed fluid and hemodynamic therapy protocols with validated tools and parameters in children need to be included in intraoperative management in this surgical intervention to guide fluid and vasoactive therapy. Monitoring mean blood pressure is not enough for optimal hemodynamic management. Fluid management is best assessed with aortic blood flow peak velocity variation with echocardiography or esophageal doppler probe (13). The limiting factor of echocardiography is expertise. Transthoracic echocardiography can be difficult to perform if the patient is in prone position which can limit access to the apex or the sternal notch and the solution can be either an esophageal doppler probe or transoesophageal echocardiography however the latter necessitates expertise. Flotrac/Vigileo has been shown to reduce blood product administration, fluid requirements, postoperative pulmonary and gastrointestinal complications and length of intensive care unit stay in spine surgery (11). Stroke volume variation with Flotrac/Vigileo can be used for fluid therapy. With this device, stroke volume variation is determined with the arterial pressure waveform analysis however evidence in children with Flotrac/Vigileo on the impact of postoperative outcome are lacking $(10,12)$. Studies in children with Flotrac/Vigileo are needed to clarify the impact on postoperative outcome.

Enhanced recovery after surgery protocols have been shown to decrease postoperative complications in scoliosis surgery in children, integrating these pathways in this surgical setting can improve postoperative evolution (3). Predictors of postoperative outcomes include patient's global status, type of surgery, emergency age and transfusion (14). Goal directed therapies are possible solutions for postoperative outcome improvement in critical ill children and in major pediatric surgery.

Minimal invasive surgical techniques such as fusionless bipolar fixation of the spine with lumbar or pelvic distal anchoring according to the etiology of scoliosis could also improve postoperative outcomes 
in this major surgery [figures 1 and 2] (16).

\section{Conclusion}

To upgrade postoperative outcome in pediatric scoliosis surgery, transfusion guided protocols with point of care tests, goal directed fluid and hemodynamic therapy with validated tools and parameters in children and enhanced recovery after surgery need to be included in this surgical setting management. Minimal invasive surgical techniques are also promising alternatives to reduce postoperative morbidity in this surgery.

\section{Declarations}

Conflict of Interest: The authors declared no conflicts of interest.

Funding: None

Presentation of preliminary results: This manuscript has been registered as a preprint under the DOI registration number https://doi.org/10.21203/rs.3.rs-765785/v1 on Research Square, a preprint platform.

\section{Authors contribution:}

Claudine Kumba conceptualized and designed the study and drafted the initial manuscript. She designed the data collection instruments, collected data, carried out initial and final analyses.

Lotfi Miladi reviewed the manuscript.

Ethics Approval: This study received approval from the Ethics Committee of Necker on 21 March 2017 under registration number 2017-CK-5-R1 and waived patient consent.

\section{References}

1) Claudine Kumba. "A Retrospective Descriptive Cohort Study of Preoperative, Intraoperative and Postoperative Management of Children in Scoliosis Surgery". EC Anaesthesia 5.2 (2019): 20-29. 
2) Kumba C, Cresci F, Picard C et al (2017) Transfusion and Morbi-Mortality Factors: An Observational Descriptive Retrospective Pediatric Cohort Study. J Anesth Crit Care Open Access 8(4): 00315. DOI :10.15406/jaccoa.2017.08.00315.

3) Kumba C, et al. Rapid Recovery Pathways after Surgery in Children: A Systematic Review and MetaAnalysis. Med J Clin Trials Case Stud 2019, 3(2): 000211. DOI: 10.23880/mjccs-16000211.

4) Kumba C, Querciagrossa S, Harte C, Willems A et al. A Systematic Review and Meta-analysis of Goal Directed Intra-Operative Transfusion Protocols Guided by Viscoelastic Methods and Perioperative Outcomes in Children. Int J Recent Sci Res $2019 ; 10$ (03), pp. 31466-31471.

5) Kumba C, Willems A, Querciagrossa S et al. A Systematic Review and Meta- Analysis of Intraoperative Goal Directed Fluid and Haemodynamic Therapy in Children and Postoperative Outcome. J Emerg Med Critical Care 2019;5(1):1-9. DOI: 10.13188/2469-4045.1000020.

6) Kumba C (2020) Physiology Principles Underlying Goal Directed Therapies in Children. Res Pediatr Neonatol. 4(4).RPN.000591.2020.Doi/10.31031/RPN.2020.04.000591.

7) Kumba C (2020) Rationale of Goal Directed Therapies in Children. Adv Pediatr Res 7:42. Doi:10.35248/2385-4529.20.7.42.

8) Kumba C (2019) “Do Goal Directed Therapies Improve Postoperative Outcome in Children? (Perioperative Goal Directed Fluid and Hemodynamic Therapy; Transfusion goal directed therapy using viscoelastic methods and enhanced recovery after surgery and Postoperative outcome): A Study Research Protocol". Acta Scientific Paediatrics 2(7) :17-19.Doi:10.31080/ASPE.2019.02.0094.

9) Kumba C (2020) Goal directed fluid and hemodynamic therapy and postoperative outcomes in children: Value of transthoracic echocardiographic aortic blood flow peak velocity variation: A multicentre randomized controlled trial protocol. Adv Pediatr Res 7:35. doi: 10.35248/2385-4529.20.7.35. 
10) Slagt C, Malagon I, Groeneveld ABJ. Systematic review of uncalibrated arterial pressure waveform analysis to determine cardiac output and stroke volume variation. British Journal of Anaesthesia 2014; 112 (4):626-37.

11) Bacchin MR, Ceria CM, Giannone S et al. Goal-Directed Fluid Therapy Based on Stroke Volume Variation in Patients Undergoing Major Spine Surgery in Prone Position. Spine 2016; 41:E1131-E1137.

12) Biais $M$, Nouette-Gaulain $K$, Roullet $S$ et al. A comparison os stroke volume variation measured by Vigileo/FloTrac System and Aortic Doppler Echocardiography. Anest Analg 2009; 109:466-9.

13) Gan H, Cannesson M, Chandler JR, Ansermino JM. Predicting fluid responsiveness in children: a systematic review. Anest Analg 2013; 117:1380-92.

14. Kumba C, Lenoire A, Cairet P, Dogaru-Dedieu E, Belloni I, Orliaguet G. Is Transfusion an Independent Risk Factor of Postoperative Outcome in Pediatric Orthopedic Surgical Patients ? A Retrospective Study. J Emerg Med Critical Care 2018 ; 4(2) :7.

15. Claudine Kumba. Liver Transplantation in Children and Impact of Intraoperative Goal Directed Therapies on Postoperative Outcome. Research Square 2021; DOI: https://doi.org/10.21203/rs.3.rs744584/v1.

16. Miladi L, Gaume M, Khouri N, Johnson M, Topouchian V, Glorion C. Minimally Invasive Surgery for Neuromuscular Scoliosis. Spine 2018; 43(16), E968-E975.

\section{Tables}

Table 1 General characteristics 


\begin{tabular}{|l|l|}
\hline Characteristic & $\mathbf{N}=\mathbf{1 1 6}$ \\
\hline Mean age \pm standard deviation in months & $147.5 \pm 40.2$ \\
\hline Median weight [interquartile range] in kilograms & $34[24-45]$ \\
\hline ASA I n (\%) & $1(0.9)$ \\
\hline ASA II n (\%) & $35(30)$ \\
\hline ASA III n (\%) & $63(54.3)$ \\
\hline ASA IV n (\%) & $17(14.7)$ \\
\hline Idiopathic scoliosis n (\%) & $45(38.8)$ \\
\hline Non idiopathic scoliosis n (\%) & $71(61.2)$ \\
\hline Emergency surgery n (\%) & $5(4.3)$ \\
\hline Elective surgery n (\%) & $111(95.7)$ \\
\hline Re-operation n (\%) & $12(10.3)$ \\
\hline Patients with intra-operative and or postoperative complications (organ \\
failure or sepsis) n (\%) & $28(24.1)$ \\
\hline Intra-operative anaphylaxis n (\%) & \\
\hline Intra-operative respiratory failure n (\%) & $1(0.9)$ \\
\hline Intra-operative hemorrhagic shock n (\%) & $1(0.9)$ \\
\hline Postoperative renal failure n (\%) & $3(2.6)$ \\
\hline Postoperative cardio-circulatory failure n (\%) & $1(0.9)$ \\
\hline Postoperative respiratory failure n (\%) & $2(1.7)$ \\
\hline Postoperative neurologic failure n (\%) & $3(2.6)$ \\
\hline Postoperative abdominal sepsis n (\%) & $7(6)$ \\
\hline Postoperative septicemia n (\%) & $2(1.7)$ \\
\hline Postoperative urinary sepsis n (\%) & $2(1.7)$ \\
\hline Postoperative surgical wound sepsis n (\%) & $3(2.6)$ \\
\hline In-hospital mortality n (\%) & $8(6.9)$ \\
\hline Transfusion n (\%) & $0(0)$ \\
\hline Median length of intensive care unit stay in days [range] & $56(48.3)$ \\
\hline Median length of hospital stay in days [range] & $4[0-25]$ \\
\hline Median total length of hospital stay in days [range] & $10[1-50]$ \\
\hline Median length of mechanical ventilation in days [range] & $12[2-67]$ \\
\hline Median packed red blood cells volume in units [range] & $0[0-21]$ \\
\hline Median fresh frozen plasma volume in units [range] & $0[0-3]$ \\
\hline Median concentrated platelet units [range] & $0[0-5]$ \\
\hline Mean preoperative hemoglobin levels \pm standard deviation in g/dL & $0[0-2]$ \\
\hline Mean postoperative hemoglobin levels \pm standard deviation in g/dL & $12.4 \pm 1.7$ \\
\hline Median crystalloid volume in ml [range] & $10.7 \pm 1.6$ \\
\hline Median colloid volume in ml [range] & $1750[500-$ \\
\hline & $4684]$ \\
\hline & $2000[0-$ \\
\hline & $6952]$ \\
\hline
\end{tabular}

Table 2 Co-morbidities 


\begin{tabular}{|c|c|}
\hline Co-morbidity & patients (\%) \\
\hline Central Core Myopathy & $1(0.9)$ \\
\hline Cerebral anoxic lesions & $22(18.9)$ \\
\hline Congenital heart disease & $3(2.6)$ \\
\hline Convulsive encephalopathy & $2(1.7)$ \\
\hline Di George Syndrome & $2(1.7)$ \\
\hline Ewing's sarcoma & $1(0.9)$ \\
\hline Goldenhar's syndrome & $1(0.9)$ \\
\hline Gorlin's syndrome & $2(1.7)$ \\
\hline Hurler's syndrome & $2(1.7)$ \\
\hline Muscular dystrophy & $2(1.7)$ \\
\hline Myelomeningocele & $4(3.5)$ \\
\hline Neurofibromatosis & $7(6.0)$ \\
\hline Osteogenesis imperfecta & $4(3.5)$ \\
\hline Pierre Robin syndrome & $2(1.7)$ \\
\hline Polymalformative syndrome & $4(3.5)$ \\
\hline Polytrauma & $2(1.7)$ \\
\hline Prader Willi syndrome & $2(1.7)$ \\
\hline Psychomotor deficiency & $3(2.5)$ \\
\hline Scoliosis & $1(0.9)$ \\
\hline Sepsis & $1(0.9)$ \\
\hline Spina Bifida & $2(1.7)$ \\
\hline Spinal muscular amyotrophy & $2(1.7)$ \\
\hline West syndrome & $1(0.9)$ \\
\hline Williams syndrome & $1(0.9)$ \\
\hline Xeroderma pigmentosum & $1(0.9)$ \\
\hline
\end{tabular}

Figures 


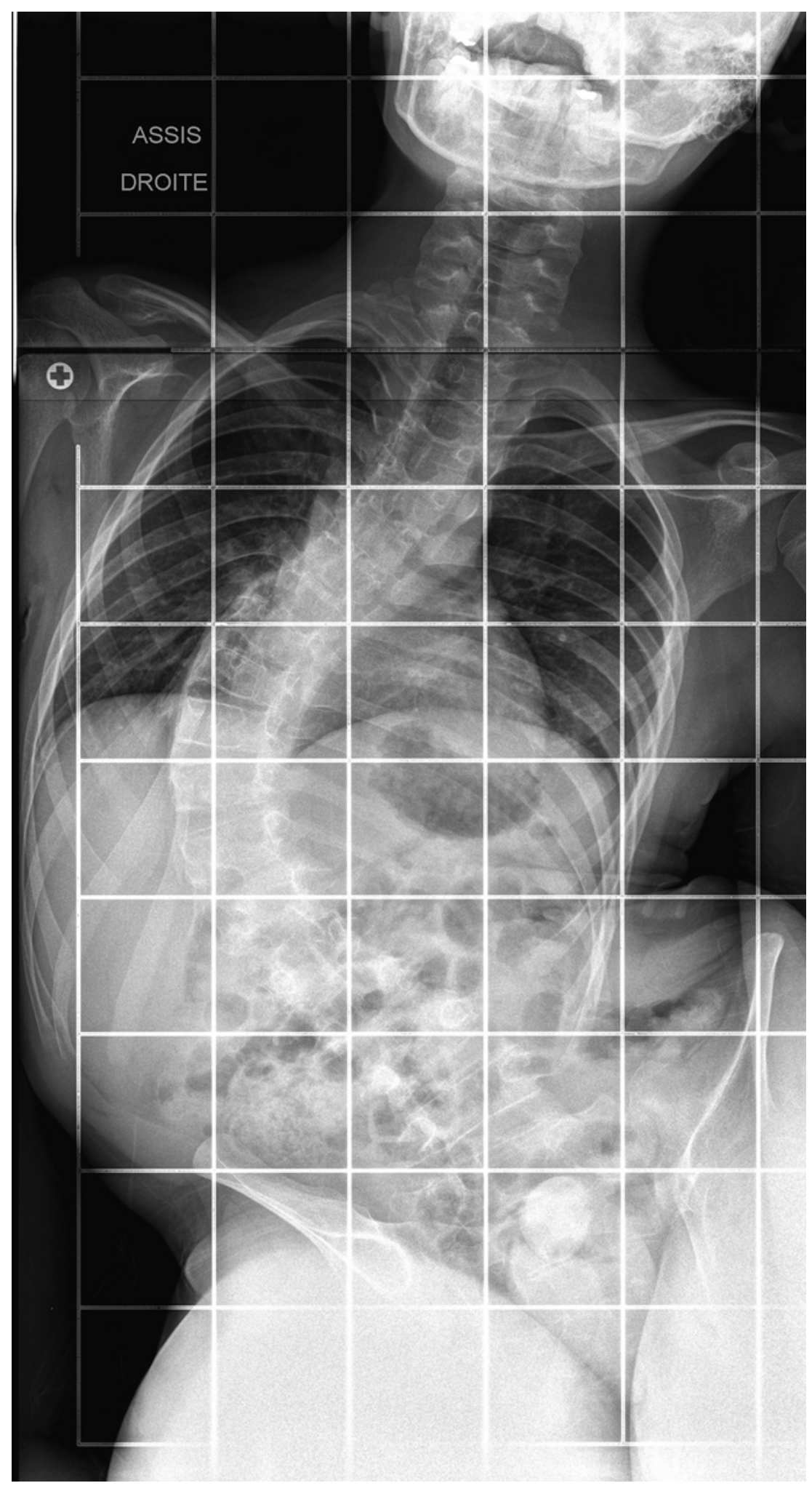

Figure 1

Preoperative spine radiography of a 13 years old child with neuromuscular scoliosis 


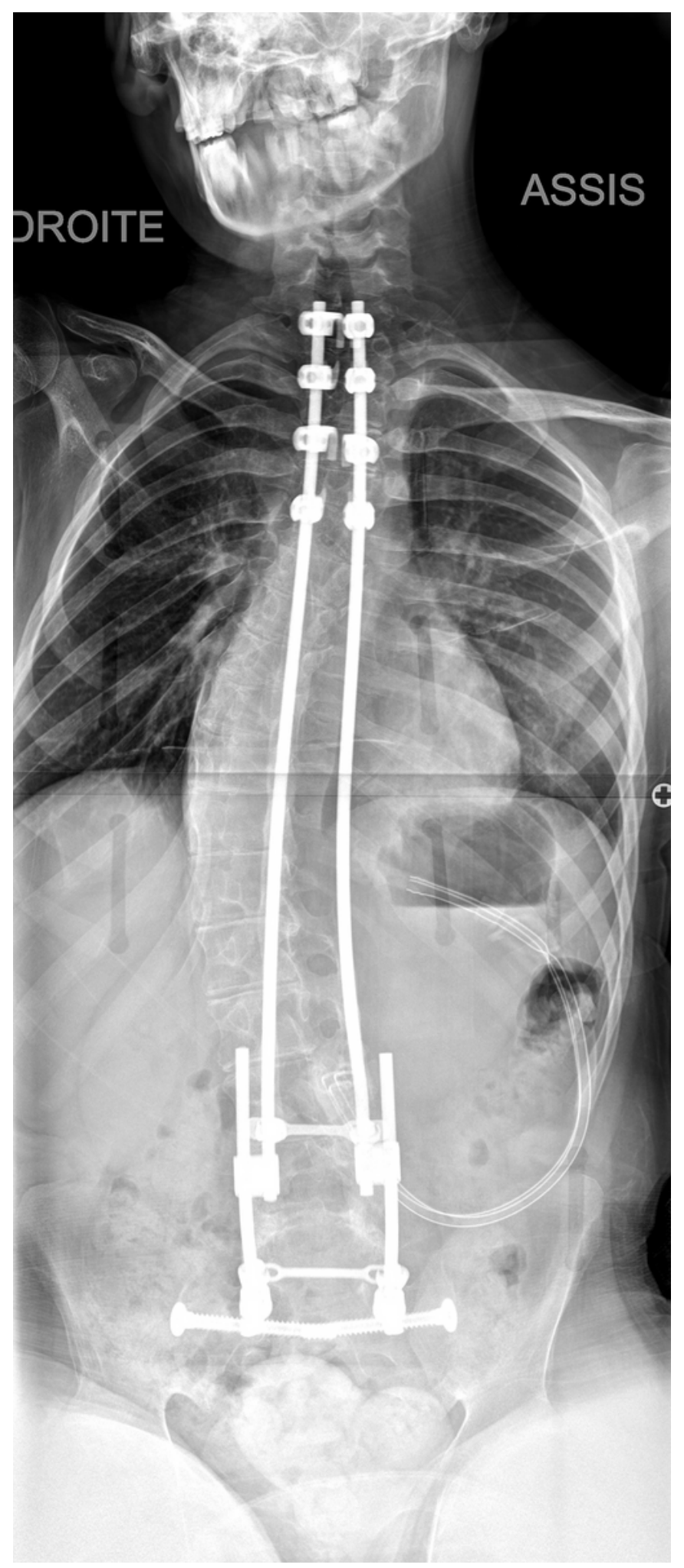

Figure 2

Postoperative spine radiography of a 13 years old child with neuromuscular scoliosis after a minimal invasive surgical technique 


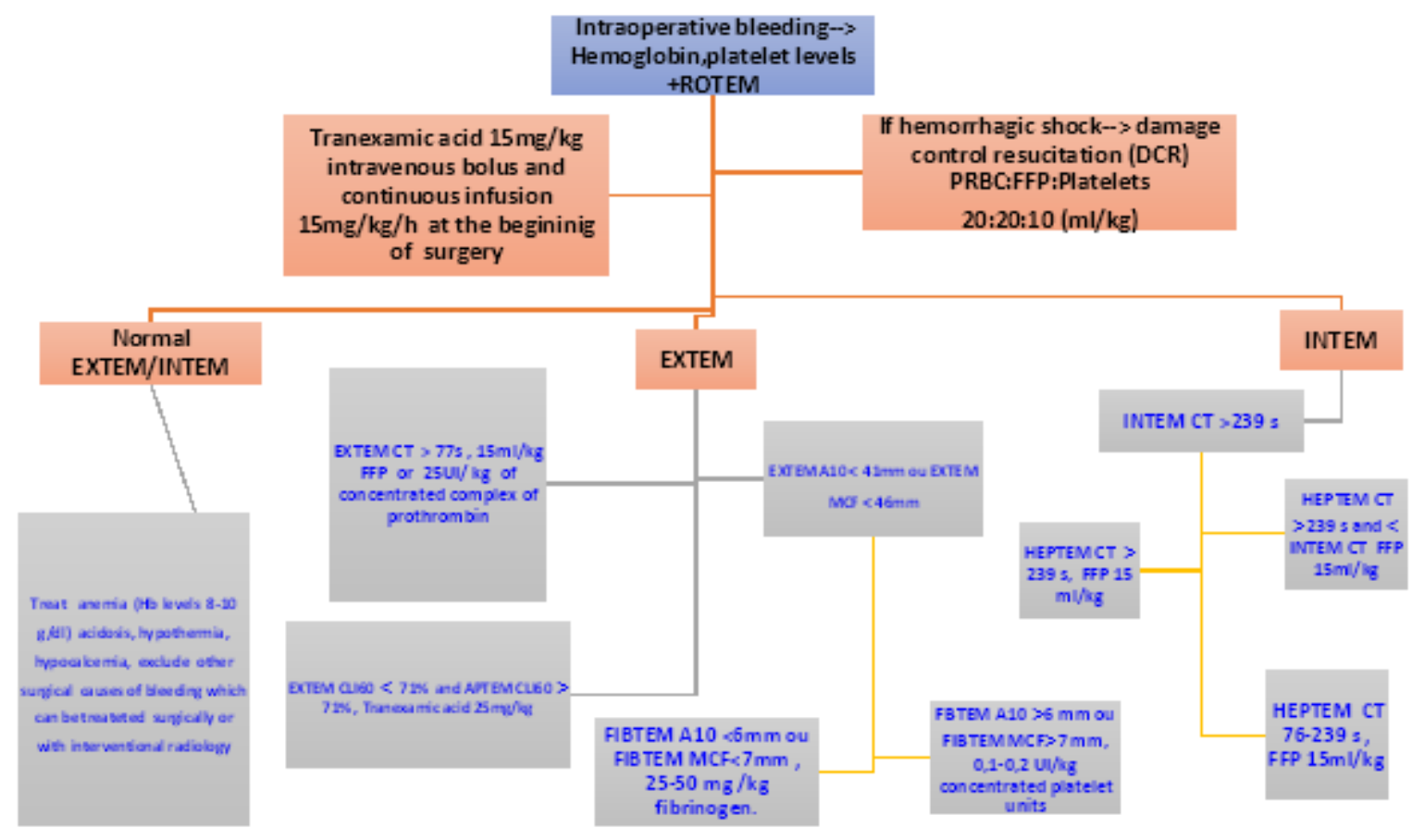

\section{Figure 3}

ROTEM Algorithm in children between enfant 0-24 months. CT=coagulation time in seconds, A10= clot firmness at 10 minutes, $\mathrm{MCF}=$ maximum clot firmness, $\mathrm{CLI} 60=$ lysis index in $\% 60$ minutes after $\mathrm{CT}, \mathrm{ML}=$ maximum lysis in \%, FFP=fresh frozen plasma, $\mathrm{PRBC}=$ packed red blood cells, $\mathrm{Hb}=$ hemoglobin

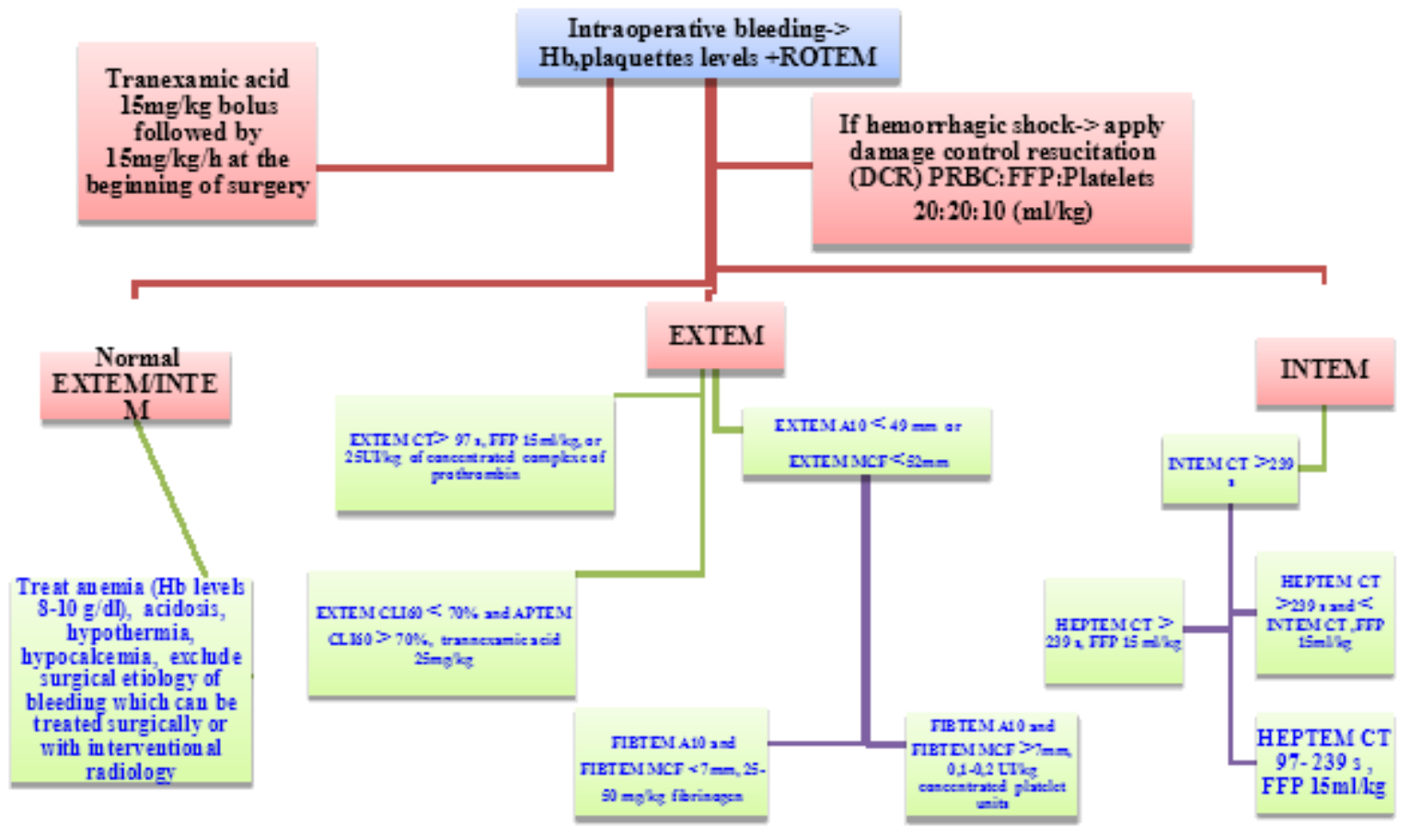

\section{Figure 4}

ROTEM Algorithm in children 2-16 years. CT=coagulation time in seconds, A10= clot firmness after 10 minutes, $\mathrm{MCF}=$ maximum clot firmness, $\mathrm{CLI60}=$ lysis index in \% 60 minutes after CT, ML= maximum lysis 
in \%, FFP=fresh frozen plasma, $\mathrm{PRBC}=$ packed red blood cells, $\mathrm{Hb}=$ hemoglobin

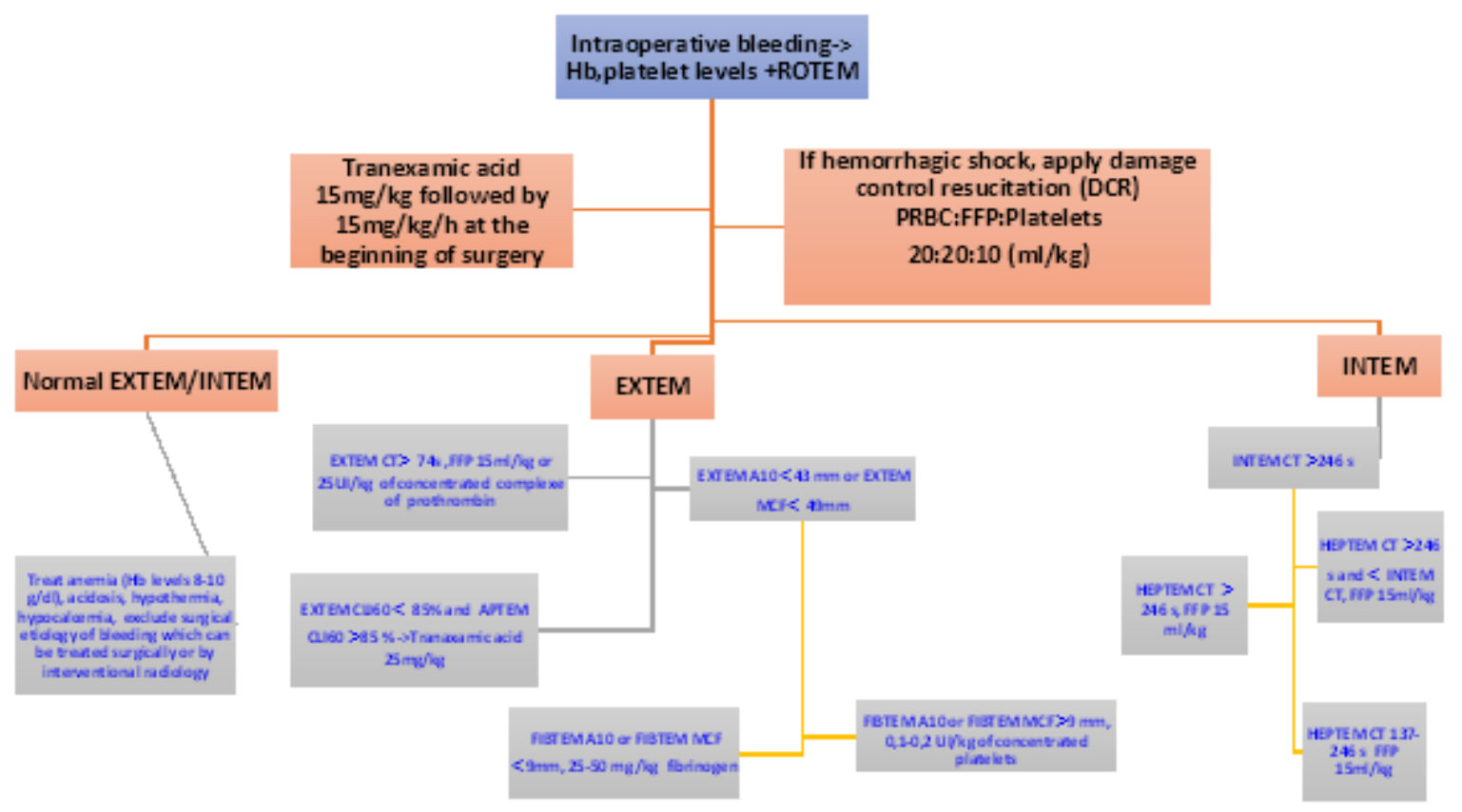

\section{Figure 5}

ROTEM Algorithm $>16$ years. $C T=$ coagulation time in seconds, $A 10=$ clot firmness at 10 minutes, MCF =maximum clot firmness, CLI60= lysis index in \% 60 minutes after CT, ML= maximum lysis in \%, $\mathrm{FFP}=$ fresh frozen plasma, $\mathrm{PRBC}=$ packed red blood cells, $\mathrm{Hb}=$ hemoglobin 